\title{
ENSINO DO DESIGN: panorama dos diferentes cursos de design no Brasil
}

SELAU, Luiza Grazziotin; Ma; Faculdade da Serra Gaúcha (FSG)

luiza.selau@fsg.br

VIEIRA, Gabriel Bergmann Borges; Me; Faculdade da Serra Gaúcha (FSG) gabriel.vieira@fsg.br

Resumo: A difusão do design no Brasil tem forte relação com a oferta de cursos de design ao longo de todo o território nacional. O ensino do design, pela natureza interdisciplinar própria da área, fomenta diversidade de cursos, relacionados diretamente com o contexto em que estão inseridos. Este estudo apresenta a identificação, agrupamento e análise dos diferentes cursos de graduação em design oferecidos por instituições públicas e privadas em território nacional, com base em dados do Ministério da Educação (MEC, 2013), por meio de consulta ao E-MEC. O trabalho mostra a distribuição dos diferentes cursos de graduação em design em todos os estados do Brasil, e ainda, com base em dados do Instituto Brasileiro de Geografia e Estatística (IBGE, 2010), é traçada uma relação entre oferta de cursos e o Produto Interno Bruto (PIB) dos estados. Como resultado apresenta a relação entre design, desenvolvimento econômico e contexto cultural.

Palavras-chave: Ensino do design, cursos de design, distribuição regional.

Abstract: The diffusion of design in Brazil has a strong relationship with offering design courses throughout the country. The design education, the interdisciplinary nature of the field itself, fosters diversity of courses directly related to the context in which they are inserted. This study presents the identification, collation and analysis of different undergraduate courses in design offered by public and private institutions in the country, based on data from the Ministry of Education (MEC, 2013) by querying the E-MEC. The work shows the distribution of different undergraduate courses in design in all states of Brazil, and, based on data from the Brazilian Institute of Geography and Statistics (IBGE, 2010), is drawn a link between the courses offered and the PIB of the states. As a result shows the relationship between design, economic development and cultural context.

Keywords: Teaching design, design courses, regional distribution. 


\section{INTRODUÇÃO}

Cursos superiores em design são oferecidos em todo o Brasil através de variadas habilitações devido, principalmente, ao caráter interdisciplinar da área tanto em termos de ensino quanto da prática profissional. Em complemento, a grande extensão do território nacional e as diferenças culturais, sociais e mercadológicas interferem tanto na concepção de projetos de design como na oferta de cursos de graduação.

Dessa forma, observa-se que cada instituição de ensino possui sua própria proposta pedagógica, organização curricular e ênfases, considerando as prerrogativas e diretrizes estabelecidas pelo Ministério da Educação (MEC) para o funcionamento dos cursos no país.

A busca pela compreensão da distribuição territorial dos cursos e as especificidades encontradas em cada região do Brasil é tópico que carece de estudos específicos. Nesse sentido, este trabalho apresenta um panorama dos cursos de design oferecidos ao longo do território nacional. Para tanto, parte de revisão teórica sobre: i) o ensino do design; ii) cursos de design e iii) modalidades de ensino.

Por meio de pesquisa na plataforma de cadastro de Instituições do Ministério da Educação, o E-MEC, foi pesquisado o número de cursos ofertados e as especificidades de cada curso. Posteriormente, partiu-se para a identificação, agrupamento e análise dos diferentes cursos de graduação em design oferecidos por instituições públicas e privadas do Brasil.

Ao considerar que as características relacionadas ao desenvolvimento econômico podem interferir na natureza dos cursos de design, este estudo apresenta, ainda, a relação entre oferta de cursos e o Produto Interno Bruto (PIB) dos estados com maior expressividade na oferta de cursos. De acordo com IBGE (2010), o produto interno bruto (PIB) representa a soma (em valores monetários) de todos os bens e serviços finais produzidos numa determinada região durante um período de avaliação.

Desta forma, percebe-se que através dos dados apresentados neste estudo é possível compreender o cenário do ensino do design do Brasil, mapear a concentração de cursos nos diferentes estados, e, oferecer subsídio para trabalhos que possam explorar as especifidades de cada curso, região e reflexo do ensino na disseminação do design no país.

\section{ENSINO DO DESIGN}

A compreensão do ensino do design, desde as primeiras escolas da área até hoje, pode partir de uma análise da distinção entre a arte e o artesanato, afinal, esta questão circundou as primeiras escolas de design na Alemanha: unir ou distanciar estas atividades. Segundo Cardoso (2008), a ideia que se tem hoje sobre artesanato é de um trabalho diferenciado e especial, mas este tipo de pensamento é fruto da industrialização, pois antes faria pouco ou nenhum sentido. A criação das escolas superiores de design, centradas na relação entre artes e artesanato tem nítido deslocamento para a separação entre projetar e fabricar, ou seja, do artesanato para o design (CARDOSO, 2008).

O fato é que o designer se limita a projetar o objeto para ser fabricado por meios mecânicos, o que comprova a visão de que o trabalho do design é diferente do trabalho do artesão após a Revolução Industrial (CARDOSO, 2008). E quanto ao surgimento do design ligado à industrialização, mesmo que ainda tenha em suas raízes 
relações fortes com o artesanato e a arte, Guimarães (2007) afirma que "o designer pode e deve contribuir para o avanço da indústria nacional", enfatizando a questão de inserir características nacionais aos artefatos resultantes de processos de design.

Moura e Somma Junior (2007) deixam claro que é preciso que o profissional do design seja consciente de seu contexto histórico, capaz de estabelecer relações para o desenvolvimento de projetos e adotar visão crítica a respeito da complexidade na qual está inserido. Com isso, Caetano (2004) observa que o design é visto como componente fundamental na satisfação do consumidor e se firma, nos mais diversos setores, no meio empresarial. Tal questão explica o atendimento da demanda por formação superior em design ocorrer através de uma ramificação de habilidades e ênfases, provenientes de projetos pedagógicos de diversas orientações (CAETANO, 2004).

As diferenças entre as ênfases se deve à atuação do designer acontecer juntamente com outros profissionais das mais variadas áreas (COUTO; OLIVEIRA, 1999) ao mesmo tempo que estabelece relações com o contexto do território cultural, tecnológico e econômico em que o curso está inserido.

O desenvolvimento de projetos com acentuado caráter interdisciplinar possibilita a integração das diversas áreas de conhecimento além de mesclar a teoria e a prática, intrínseca da atividade do design. Cabe ressaltar que, mesmo buscando conhecimento e informações em diversos campos para sua atuação e afirmação profissional, quando se ensina design, a atividade projetual é uma constante que ocorre tanto em disciplinas isoladas específicas quanto em trabalhos interdisciplinares.

\section{CURSOS DE DESIGN}

Quanto à situação dos cursos de design, de acordo com o Ministério da Educação (MEC; 2013), com base na plataforma de busca E-MEC que contém as Instituições de Educação Superior e os cursos cadastrados no MEC, atualmente no Brasil são ofertados entre 500 e 600 cursos de design. Esses dados não são precisos pois a plataforma de busca do site disponibiliza mais de um formato de pesquisa, e seus resultados não são iguais.

A diferença, provavelmente, é decorrência das Instituições de Ensino Superior que estão com os cursos "em extinção" e, como consequência, em uma das buscas, estes cursos são computados como "em funcionamento", enquanto, em outra, por estarem "em extinção", não são considerados, sendo excluídos do resultado.

Outra hipótese é que algumas Instituições de Ensino Superior (IES), possam estar cadastradas com cada habilitação que oferecem, aparecendo uma vez na primeira busca e, na outra, aparecendo mais vezes em decorrência do número de habilitações que oferecem.

O maior grupo de cursos é oferecido apenas com a nomenclatura de design, sem habilitação específica. Estes cursos graduam profissionais com formação em design. Alguns cursos de design oferecem "linhas de formação" de forma que o diploma qualifica o aluno como graduado em design, não fazendo menção à ênfase escolhida pelo acadêmico (E-MEC, 2013).

\subsection{Modalidades}

De quase 600 cursos superiores de design reconhecidos pelo MEC no Brasil, pode-se encontrar cursos de bacharelado, licenciatura, tecnológico e sequencial. 
Os cursos bacharelados são para quem pretende atuar de forma ampla no mercado de trabalho. Já, os cursos de licenciatura são para a formação de professores de ensino fundamental e médio, o que direciona as disciplinas a serem mais voltadas à didática, psicologia e pedagogia (VALLEI, 2012).

Por outro lado, os cursos tecnológicos, como os de bacharelado para quem pretende atuar no mercado de trabalho, além de sua curta duração (cerca de 2 ou 3 anos), possuem foco específico (o curso é direcionado à uma parte específica de alguma área do conhecimento). Os cursos tecnológicos são focados na inovação e nas necessidades da sociedade e, por fim, são direcionados ao setor produtivo, na maioria das vezes, voltados ao mercado local, visando aplicação prática (VALLEI, 2012).

Os cursos sequenciais são cursos superiores de curta duração (cerca de 2 anos) e assim como os tecnológicos oferecem habilidades específicas em determinadas áreas. A característica mais notável dos sequenciais é que ao final o estudante possui um diploma de nível superior e só pode ingressar em especialização de pós-graduação - Lato-Sensu, não tendo acesso à mestrado ou doutorado - Stricto-Sensu (BRASIL, 2013).

Outra questão encontrada nas ofertas de curso superior em design no país, são as opções entre aulas presenciais ou à distância (EaD - Educação à Distância). Nas aulas presenciais, os alunos e o(s) professor(es) precisam se encontrar em determinado local e horário para que as aulas aconteçam. Nas aulas à distância, isso não é necessário (exceto para as avaliações) - o que possibilita que os alunos organizem seus próprios horários, de acordo com suas disponibilidades (VALLEI, 2012).

Quanto à avaliação, frequência e custos de aulas presenciais ou à distância, existem algumas diferenças, conforme figura 1.

\begin{tabular}{|c|c|c|}
\hline & Cursos EaD & Cursos Presenciais \\
\hline Aulas & Online ou via satélite & Presenciais \\
\hline Avaliação & $\begin{array}{l}\text { Provas presenciais (Mesmo a distância, } \\
\text { o Ministério da Educação exige que as } \\
\text { avaliações sejam feitas na instituição) }\end{array}$ & Provas presencias \\
\hline Horários & Flexíveis & Fixos \\
\hline Frequência & Contabilizada por atividades e trabalhos & $\begin{array}{l}\text { Contabilizada pela } \\
\text { presença do aluno em } \\
\text { sala }\end{array}$ \\
\hline Custos & $\begin{array}{l}\text { Aluno economiza entre } 10 \text { e } 15 \% \text { nas } \\
\text { mensalidades }{ }^{*}\end{array}$ & $\begin{array}{l}\text { Além da mensalidade, } \\
\text { aluno precisa gastar com } \\
\text { transporte e } \\
\text { alimentação }\end{array}$ \\
\hline
\end{tabular}

Figura 1: Diferenças entre curso EaD e Presencial

Fonte: Vallei (2012)

$\mathrm{Na}$ área do design, a maior parte dos cursos é disponibilizada no formato de aulas presenciais, (apenas três cursos de formação EaD foram identificados, sendo os três tecnológicos).

Com base nos dados do E-MEC (2013), no Brasil são oferecidos cerca de 316 cursos Tecnológicos, 257 cursos de Bacharelado, 4 sequenciais e 1 de licenciatura (em design de moda). 
Os cursos são ofertados por instituições públicas ou privadas, sendo que as públicas "são mantidas pelo Poder Público, na forma Federal, Estadual ou Municipal" e por serem "financiadas pelo Estado, não cobram matrícula nem mensalidade" (MINISTÉRIO DAS RELAÇÕES EXTERIORES, 2013). As privadas "são administradas por pessoas físicas ou jurídicas de direito privado, com ou sem finalidade de lucro" (MINISTÉRIO DAS RELAÇÕES EXTERIORES, 2013). Dentre os cursos de design no Brasil, 509 em são oferecidos por Instituições Privadas e 69 são oferecidos em Instituições Públicas.

\section{MÉTODO}

Com o intuito de alcançar os objetivos descritos anteriormente, o método empregado para este estudo consiste em pesquisa de abordagem descritiva que busca de forma geral descrever características, identificar possíveis variáveis e descobrir a existência de associações entre as varáveis (GIL, 2010).

O método de trabalho adotado apresenta as seguintes etapas:

Quadro 1: método de trabalho adotado

\begin{tabular}{|l|l|l|l|}
\hline ETAPA DA PESQUISA & OBJETIVO & MÉTODO DE COLETA & MÉTODO DE ANÁLISE \\
\hline Mapeamento dos cursos & $\begin{array}{l}\text { Identificação dos } \\
\text { diferentes cursos de } \\
\text { design ofertados no } \\
\text { Brasil e a distribuição } \\
\text { regional dos mesmos. }\end{array}$ & $\begin{array}{l}\text { Pesquisa realizada no } \\
\text { E-MEC. }\end{array}$ & $\begin{array}{l}\text { Seleção, agrupamento e } \\
\text { classificação de dados } \\
\text { por meio do software } \\
\text { Excel. } \\
\text { Apresentação dos dados } \\
\text { em matriz de relação e } \\
\text { gráfico de área. }\end{array}$ \\
\hline $\begin{array}{l}\text { Mapeamento do PIB dos } \\
\text { Estados }\end{array}$ & $\begin{array}{l}\text { Identificação o } \\
\text { Produto Interno Bruto } \\
\text { (PIB) de cada estado } \\
\text { do Brasil. }\end{array}$ & $\begin{array}{l}\text { Pesquisa realizada na } \\
\text { base de dados do IBGE. }\end{array}$ & $\begin{array}{l}\text { Seleção, agrupamento e } \\
\text { classificação de dados } \\
\text { por meio do software } \\
\text { Excel. }\end{array}$ \\
\hline $\begin{array}{l}\text { Análise de dados de } \\
\text { Cursos x PIB }\end{array}$ & $\begin{array}{l}\text { Identificar relaçães } \\
\text { entre a oferta de } \\
\text { cursos e Produto } \\
\text { Interno Bruto (PIB) } \\
\text { dos estados mais } \\
\text { expressivos em } \\
\text { número de cursos. }\end{array}$ & $\begin{array}{l}\text { Utilização de dados } \\
\text { coletados nas etapas } \\
\text { anteriores. }\end{array}$ & $\begin{array}{l}\text { Seleção, agrupamento e } \\
\text { classificação de dados } \\
\text { por meio do software } \\
\text { Excel. }\end{array}$ \\
\hline
\end{tabular}

Fonte: Elaborado pelos autores, com base na pesquisa realizada.

A primeira etapa, com o objetivo de identificar os diferentes cursos ofertados no Brasil, utilizou-se como fonte de dados no site do E-MEC (2013), plataforma do Ministério da Educação que permite a tramitação eletrônica de processos de regulamentação de IES's pela internet (E-MEC, 2013) e disponibiliza informações sobre as IES's cadastradas, conforme já mencionado, a fim de identificar quantas instituições oferecem o curso de design no Brasil, verificando estado por estado e as habilitações disponíveis.

Os dados foram selecionados, agrupados e classificados por meio por meio da utilização do software Excel, considerando as seguintes categorias: tipo de curso; número de cursos e estado. 
Após essa etapa, utilizando como fonte de informação dados do IBGE (2013), foram compilados os indicadores de cada estado relacionados ao Produto Interno Bruto (PIB), agrupados e classificados por estado por meio do software Excel.

Após coleta desses dados, partiu-se para a análise e cruzamentos entre os dados do E-MEC (2013) e dados do IBGE (2010), buscando identificar relações entre o PIB e número de cursos de design em nos estados com maior representatividade absoluta.

\section{DESCRIÇÃO E ANÁLISE DOS RESULTADOS}

Com base em dados do E-MEC (2013), quanto aos cursos oferecidos no Brasil, foram identificadas 18 habilitações: Design; Design Gráfico; Design de Produto; Design de Moda; Design de Interiores; Design Digital; Design Industrial; Animação; Design de Calçados; Design de Móveis; Design de Embalagens; Design de Games; Programação Visual; Design Publicitário; Design de Carnaval; Design de Ambientes; Design de Jóias e Gemas.

A tabela apresenta os dados obtidos, demonstrando que em todo o Brasil 18 habilitações são oferecidas para o curso superior de design nos 26 estados mais 0 Distrito Federal, resultando em 500 cursos (desconsiderando os em extinção ou inativos).

Tabela 1: Mapeamento de cursos por região por meio de mapa de área.

\begin{tabular}{|c|c|c|c|c|c|c|c|c|c|c|c|c|c|c|c|c|c|c|c|c|c|c|c|c|c|c|c|c|}
\hline & $\approx$ & $u$ & $\stackrel{\Upsilon}{a}$ & n & $\vec{\sim}$ & 出 & $\stackrel{৩}{\Sigma}$ & $\sum^{n}$ & $\stackrel{\llcorner}{\Sigma}$ & ㅇ & 㟔 & $\overleftarrow{\infty}$ & 岕 & $\vec{\alpha}$ & w & 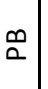 & $z_{\propto}$ & 剀 & $\bar{\alpha}$ & 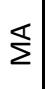 & 인 & 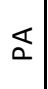 & ○ & U & $\sum_{<}$ & $\stackrel{\propto}{\propto}$ & $\frac{a}{<}$ & 胥 \\
\hline DESIGN & 15 & 16 & 6 & 39 & 8 & 4 & 9 & 2 & & 2 & 1 & 7 & 1 & 1 & 3 & 2 & 1 & 2 & 1 & 2 & & 2 & & & 3 & & 2 & 129 \\
\hline GRÁFICO & 9 & 6 & 8 & 24 & 7 & 3 & 9 & 3 & 2 & 7 & 4 & 2 & 1 & 3 & 5 & 2 & 1 & 5 & 2 & 1 & 1 & 3 & 2 & 1 & 3 & 3 & & 117 \\
\hline PRODUTO & 10 & 6 & 4 & 10 & 1 & 2 & 3 & & & & & & & & 1 & & & 3 & & & & 1 & & & & & & 41 \\
\hline MODA & 11 & 10 & 9 & 27 & 4 & 3 & 10 & 1 & 1 & 5 & 5 & 5 & & 1 & 6 & 1 & & 5 & 1 & 1 & & 1 & & & 1 & & & 108 \\
\hline INTERIORES & 6 & 8 & 9 & 24 & 4 & 2 & 7 & 1 & 2 & 3 & 3 & 3 & 1 & 2 & 4 & 2 & 1 & 1 & 2 & 1 & & 1 & & & 2 & & & 89 \\
\hline DIGITAL & 1 & & & 3 & & & & & & & & & & & & & & & & & & & & & & & & 4 \\
\hline INDUSTRIAL & & 1 & & & & & & & & & & & & & & & & & & & & & & & & & & 1 \\
\hline ANIMAÇÃO & & 1 & & & & & & & & & & & & & & & & & & & & & & & & & & 1 \\
\hline CALÇADOS & & 1 & & & & & & & & & & & & & & & & & & & & & & & & & & 1 \\
\hline MÓVEIS & & & 1 & & & & & & & & & & & & & & & & & & & & & & & & & 1 \\
\hline EMBALAGENS & & & & 1 & & & & & & & & & & & & & & & & & & & & & & & & 1 \\
\hline GAMES & & & & 1 & & & & & & & & & & & & & & & & & & & & & & & & 1 \\
\hline PROG. VISUAL & & & & 1 & & & & & & & & & & & & & & & & & & & & & & & & 1 \\
\hline PUBLICITÁRIO & & & & 1 & & & & & & & & & & & & & & & & & & & & & & & & 1 \\
\hline CARNAVAL & & & & & 1 & & & & & & & & & & & & & & & & & & & & & & & 1 \\
\hline AMBIENTES & & & & & & & 1 & & & 1 & & & & & & & & & & & & & & & & & & 2 \\
\hline JÓIAS E GEMAS & & & & & & & & & & 1 & & & & & & & & & & & & & & & & & & 1 \\
\hline TOTAL/ESTADO & 52 & 49 & 37 & 131 & 25 & 14 & 39 & 7 & 5 & 19 & 13 & 17 & 3 & 7 & 19 & 7 & 3 & 16 & 6 & 5 & 1 & 8 & 2 & 1 & 9 & 3 & 2 & 500 \\
\hline
\end{tabular}

Fonte: Elaborado pelos autores, com base em pesquisa realizada.

Como é possível perceber, todos os estados oferecem pelo menos um curso de design de nível superior. O único curso presente em todos os estados do Brasil é o de Design Gráfico. O curso mais ofertado é o de Design (sem habilitação específica na nomenclatura) e os outros três cursos com maior incidência no país são respectivamente: Design de Moda, Design de Interiores e Design de Produtos. 0 Estado de São Paulo apresenta o maior número de cursos, totalizando 130 ofertas de 
curso superior em Design e o estado que oferece apenas um curso é Roraima, ofertando a graduação de Design Gráfico.

Conforme exposto na Tabela 1, a quantidade de cursos por enfoque representa maior volume em cinco cursos principais: Design (129), Design Gráfico (117), Design de Moda (108), Design de Interiores (89) e Design de Produtos (41). Outras ocorrências, com incidência inferior a cinco cursos no Brasil são: Design Digital, Design de Games e Web Design.

Com base nos resultados expostos na Tabela 1, partiu-se para a criação de um gráfico de dispersão e área, para visualização da distribuição dos cursos em relação aos estados do Brasil. Dessa forma, os números referentes à quantidade de cursos foram substituídos por áreas correspondentes.

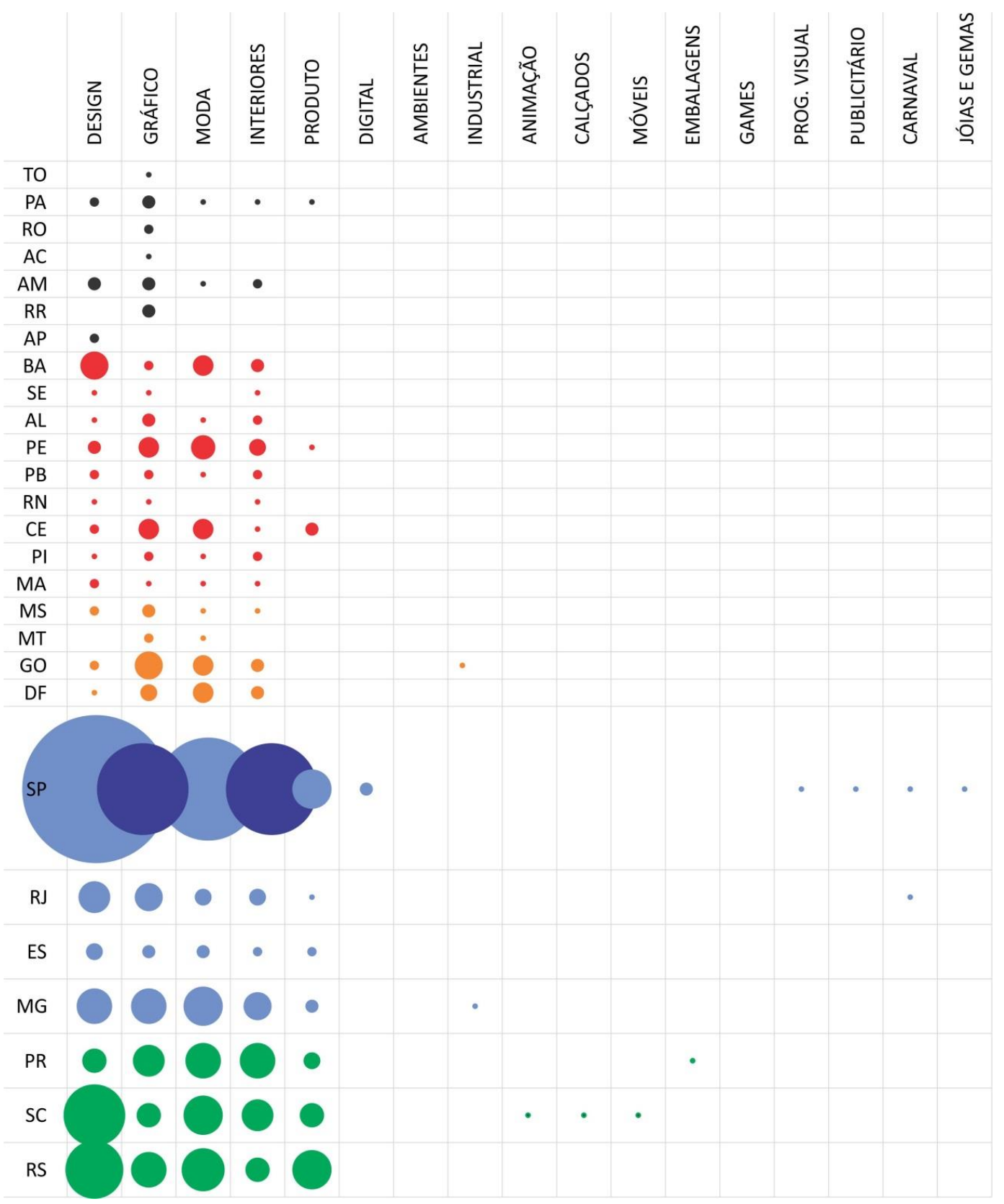

Figura 2: Gráfico de área - cursos de design $X$ estados BR

Fonte: Elaborado pelos autores, baseado em dados do E-MEC (2013) 
Ao observar a figura 1, constata-se que os estados de São Paulo, Rio Grande do Sul, Santa Catarina, Minas Gerais, Paraná e Rio de Janeiro são os que mais ofertam cursos superiores em design. Da mesma forma, percebe-se visualmente a concentração dos Cursos de Design (sem habilitação especificada), Design Gráfico, Design de Moda, Design de Interiores e Design de Produtos.

Ao observar a concentração dos cursos na Região Sul e Sudeste, é importante relacionar os dados absolutos encontrados com características dos diferentes estados. Nesse sentido, para estabelecer relação de oferta de cursos de design e dados da atividade econômica de cada estado, optou-se por coletar indicadores referentes ao Produto Interno Bruto dos estados brasileiros.

De acordo com os dados absolutos do número de cursos por estado, foi elaborado um ranking dos seis estados com o maior número de cursos de design.

Tabela 2: Ranking de cursos por estado

\section{CURSOS DE DESIGN POR ESTADO}

\begin{tabular}{l|l|l}
\hline 10 & São Paulo & 131 \\
\hline 20 & Rio Grande do Sul & 52 \\
\hline 3o & Santa Catarina & 49 \\
\hline 4o & Minas Gerais & 39 \\
\hline $\mathbf{5 0}$ & Paraná & 37 \\
\hline $\mathbf{6 0}$ & Rio de Janeiro & 25 \\
\hline & \% em relação ao total de cursos & $67 \%$ \\
\hline
\end{tabular}

Fonte: Elaborado pelos autores, baseado em dados do E-MEC (2013)

Com base na Tabela 2, além do destacado número de cursos existentes em São Paulo, observa-se a elevada participação dos estados do Rio Grande do Sul e Santa Catarina na oferta de cursos de design. Considerando que São Paulo, Rio Grande do Sul, Santa Catarina, Minas Gerais, Paraná e Rio de Janeiro são, nesta ordem, os estados com o maior número de cursos, representando $67 \%$ da oferta de cursos superiores de design no Brasil, é importante analisar o PIB dos estados do país. Nesse sentido, com base em dados do IBGE (2010), é apresentado o ranking dos seis estados de acordo com o PIB:

Tabela 3: Ranking de PIB por estado

\begin{tabular}{|c|c|c|}
\hline \multicolumn{3}{|c|}{ PIB (BILHÕES) POR ESTADO 2010} \\
\hline 10 & São Paulo & 400 \\
\hline 20 & Rio de Janeiro & 407 \\
\hline 3은 & Minas Gerais & 351 \\
\hline 40 & Rio Grande do Sul & 252 \\
\hline 50 & Paraná & 217 \\
\hline 60 & Santa Catarina & 152 \\
\hline & $\%$ do PIB Nacional (2010) & $71 \%$ \\
\hline
\end{tabular}

Com estes dados, é possível constatar que os seis estados que respondem por $67 \%$ da oferta de cursos superiores em design no Brasil apresentam o PIB mais expressivo dentre todos os estados do país, respondendo pelo percentual de $71 \%$ do 
PIB Nacional, com base no IBGE (2010). Baseando-se nestes indicadores, observa-se a relação direta entre a oferta de cursos, a atividade econômica e nível de riqueza de uma região. O estado do Rio de Janeiro, o sexto colocado quanto à oferta de cursos de design, apresenta o segundo maior PIB do Brasil. Em contrapartida, Santa Catarina, em sexto lugar quanto ao PIB apresenta o terceiro maior número de cursos de design do país. Cabe ressaltar que essa análise faz menção ao total de cursos existentes e não ao número de vagas ofertadas.

É importante destacar que o PIB tem estreita relação com o acesso aos serviços e, dessa forma, estes estados viabilizam maior oferta de cursos superiores em design. Por outro lado, ao considerar o design como atividade de desenvolvimento de produtos ou serviços focado no bem estar da sociedade, fica evidente a contrapartida e importância do design nos estados de maior concentração de cursos de graduação.

\section{CONSIDERAÇÕES FINAIS}

A natureza interdisciplinar do design torna o ensino superior diversificado em relação às suas habilitações, apresentando ênfases diversas em um território nacional tão extenso como o Brasil.

De acordo com os dados coletados e analisados, pode-se perceber que todos os estados do Brasil ofertam cursos superiores em design, sendo o maior recorte sem habilitação definida. O curso de Design Gráfico marca presença em todos os estados e as habilitações de design de moda, interiores e produto tem peso relevante no número total de cursos ofertados pelo país. Essas relações podem ser facilmente observadas por meio do gráfico de dispersão e área elaborado.

Com base no ranking de cursos por estado e PIB por estado, é fácil a observação de que os estados com maior Produto Interno Bruto e de maior desenvolvimento econômico respondam, também, pelo maior número de cursos de design.

Este estudo, ao apresentar dados preliminares que buscam relacionar o PIB com a maior concentração de cursos de graduação nos estados de São Paulo, Rio Grande do Sul, Santa Catarina, Paraná e Rio de Janeiro, destaca a importância da contextualização do ensino do design, levando em consideração, entre outras variáveis, indicadores socioeconômicos.

Para uma análise mais aprofundada, é importante considerar outras relações, tais como: i) Número de vagas ofertadas em cursos de design por estado; ii) Renda per capita e a relação com a presença e natureza de cursos; iii) Relação de Instituições Públicas e Privadas por estado; iv) Ranking de instituições por número de vagas ofertadas. 


\section{REFERÊNCIAS}

BRASIL. Cursos sequenciais. Disponível na internet em

<http://www.brasil.gov.br/empreendedor/capacitacao/cursos-sequenciais> Acesso em 23 jun. 2013.

CAETANO, Júlio Cesar. Que designers estamos formando? In: MAGUALHÃES, Eliane; et AL. Pensando Design. Porto Alegre: Uniritter, 2004.

CARDOSO, Rafael. Uma introdução à história do design. 3. ed. São Paulo: E. Blucher, 2008. 273 p.

COUTO, R.; OLIVEIRA, A. J. Formas de Design. Rio de Janeiro: 2AB/PUC-Rio, 1999.

E-MEC. Instituições de Educação Superior e Cursos Cadastrados. Disponível na internet em: <http://emec.mec.gov.br/> Acesso em 23 jun. 2013.

GIL, Antonio Carlos. Como elaborar projetos de pesquisa. São Paulo: Atlas, 2010.

GUIMARÃES,Lia Buarque de Macedo. Pela Sustentabilidade de um Design Brasileiro.

In: VAN DER LINDEN, Julio Carlos de Souza. (org.) Revista D: design, educação, sociedade e sustentabilidade. Porto Alegre: Uniritter, p. 101 - 117, 2007.

IBGE. Estados@. Censo 2010. Disponível na internet em:

<http://www.ibge.gov.br/estadosat/>. Acesso em 16 abr. 2014.

MINISTÉRIO DAS RELAÇÕES EXTERIORES. Denominações das Instituições de Ensino

Superior (IES). Disponível na internet em:

<http://www.dce.mre.gov.br/nomenclatura_cursos.html> Acesso em: 23 jun. 2013. MOURA, Monica; SOMMA JUNIOR, Nelson. Design: Interdisciplinaridade no Ensino e na Aprendizagem In: VAN DER LINDEN, Julio Carlos de Souza. (org.) Revista D: design, educação, sociedade e sustentabilidade. Porto Alegre: Uniritter, p. 65 - 79, 2007.

VALLEI, C. Curso a distância (EaD) ou presencial? In: e-Guia do Estudante - Abril. 2012. Disponível na internet em: <http://guiadoestudante.abril.com.br/vestibularenem/curso-distancia-ead-ou-presencial-697316.shtml> Acesso em: 23 jun. 2012.

Bacharelado ou Licenciatura? In: e-Guia do Estudante - Abril. 2012.

Disponível na internet em: <http://guiadoestudante.abril.com.br/vestibularenem/bacharelado-ou-licenciatura-697319.shtml> Acesso em: 23 jun. 2013.

Curso tecnológico ou tradicional? In: e-Guia do Estudante - Abril. 2012.

Disponível na internet em: <http://guiadoestudante.abril.com.br/vestibularenem/curso-tecnologico-ou-tradicional-697322.shtml> Acesso em: 23 jun. 2013. 